\title{
Evidencias de sobrepesca y mal manejo del Callinectes sapidus (Rathbun, 1896) en el lago de Maracaibo, Venezuela
}

\section{Evidence of overfishing and poor management of Callinectes sapidus (Rathbun, 1896) in Lake Maracaibo, Venezuela}

\author{
Misael Molina Molina ${ }^{1 *}$ y David Mejías Yedra ${ }^{1}$
}

\begin{abstract}
RESUMEN
El trabajo se realizó entre los años 2015 y 2018. Su objetivo fue evaluar el efecto del manejo de la pesquería del cangrejo azul sobre la sostenibilidad del recurso y aportar bases para la adopción de medidas correctivas. Esta cambió a partir de 2002, cuando los pescadores dejaron de pescar con nasas y empezaron a hacerlo con palangres; ese hecho determinó un aumento abrupto en el éxito de captura y una reducción significativa en la selectividad por talla. Se evidenció una disminución paulatina del ancho del caparazón y extracción de individuos inmaduros; el crecimiento $\left(L_{\infty}\right)$ es inferior con respecto a las pesquerías de la bahía de Chesapeake, bahía de Delaware, golfo de México y bahía de Chetumal y la mortalidad por pesca $\mathrm{F}$ es significativamente alta. La tasa de explotación E de 0.75 indica sobrepesca. Los pescadores no respetan a cabalidad la legislación vigente, no reciben apoyo financiero, enfrentan inseguridad personal y esta actividad cada día aporta menos a la economía familiar, mientras que el Estado ha fallado en el registro de datos, en la evaluación del hábitat y en la gestión del recurso. Este coctel de elementos ha condicionado su actual sobreexplotación y amenaza.
\end{abstract}

Palabras clave: gestión, legislación, mortalidad por pesca, sostenibilidad, talla

\begin{abstract}
This research was conducted between 2015 and 2018. The objective was to evaluate the effect of blue crab fishery management on the resource sustainability and provide a base for the implementation of corrective measures. Blue crab fishery changed in 2002 when fishermen stopped using fish traps and began using longlines, which rapidly increased successful fishing and significantly reduced size selectivity. A gradual reduction of carapace width and the extraction of immature individuals were evident; growth $\left(\mathrm{L}_{\infty}\right)$ is lower compared to fishery in the Chesapeake,
\end{abstract}

1 Grupo de Investigaciones en Acuicultura y Zoología Aplicada. Universidad Nacional Experimental Sur del Lago, Santa Bárbara de Zulia, Venezuela¹.molinam@unesur.edu.ve*,mejiasd@unesur.edu.ve 
Delaware, and Chetumal Bays, and the Gulf of Mexico, and fishing mortality (F) was significantly high. The exploitation rate (E) equivalent to 0.75 indicates overfishing. Fishermen do not fully respect the current legislation, do not receive financial support, and face personal insecurity. This activity contributes every day less to the family economy. In addition, the government has failed to register data, assess the habitat, or manage the resource. This combination of elements has conditioned current overexploitation and threatened this species of crustacean.

Keywords: management, legislation, fishing mortality, sustainability, size

\section{INTRODUCCIÓN}

El lago de Maracaibo está conectado al mar Caribe del que recibe agua salada, lo que le otorga su condición de estuario. Es una depresión rodeada por el oeste por la sierra de Perijá y por el este por la cordillera de Mérida. Su espejo de agua es de unos $12 \mathrm{mil} \mathrm{km}{ }^{2}$ (Buonocore et al. 2011).

El clima es cálido y húmedo y está determinado por la entrada de los vientos alisios que soplan desde el noreste y entran por el golfo de Venezuela. La época seca ocurre entre diciembre y abril. Durante los meses de mayo a octubre los alisios se debilitan dando paso a la época de lluvias (Mascialgiolli \& Febres, 2000).

La temperatura media mensual del aire es de $28^{\circ} \mathrm{C}$ (Buonocore et al. 2011). La temperatura del agua presenta variaciones diarias y estacionales influenciadas por la profundidad del lago, que disminuye $2^{\circ} \mathrm{C}$ por cada $15 \mathrm{~m}$. La mayor profundidad del estuario se encuentra en su porción oriental y no sobrepasa los $35 \mathrm{~m}$. Las temperaturas más altas se registran en junio y las más bajas en enero (Buonocore et al. 2011).

Las mareas son diurnas y débiles y las corrientes predominantes circulan en sentido levógiro (Buonocore et al. 2011), lo que está definido por el curso de los vientos al chocar contra la sierra de Perijá y la cordillera de Mérida.

El lago de Maracaibo es un sistema oligohalino (0.5-5 PSU), aunque la salinidad aumenta hasta más de 40 PSU al norte del golfo de Venezuela producto de la entrada de aguas del mar Caribe, y es mínima al sur (menos de 0.5 PSU), donde desembocan los ríos Guasare, Sosuy, Cachirí, Limón, Palmar, Apón, Negro, Santa Ana, Catatumbo, Chama, Tarra, Zulia, Escalante, Tucaní, Torondoy, Motatán, Misoa y Machango (Buonocore et al. 2011).

En el lago habitan seis especies de cangrejos del género Callinectes, a saber, el cangrejo azul Callinectes sapidus, el cangrejo moro Callinectes bocourti, el cangrejo gris Callinectes ornatus, el cangrejo sirí Callinectes danae, el cangrejo rugoso Callinectes exasperatus y el cangrejo de máscara Callinectes larvatus (Taissoun, 1969; 
Casler et al. 2011b), pero es C. sapidus la especie dominante en las capturas con más del $90 \%$ del total.

C. sapidus es un crustáceo decápodo, omnívoro y epibentónico (Williams, 1974), perteneciente a la familia Portunidae, que agrupa a los cangrejos nadadores. Esta especie se distingue por el color azul brillante de su área frontal, especialmente a lo largo de sus quelípedos; el resto de su cuerpo es de color marrón oliváceo. Su quinto par de patas están modificadas en forma de remo para nadar ágilmente.

Los cangrejos de la familia Portunidae habitan en estuarios tropicales poco profundos (Taissoun, 1969; Williams, 1974), con C. sapidus distribuido desde Nueva Escocia en Canadá, hasta el río de la Plata en Argentina (Williams, 1974; Rathbun, 1986); aunque para el último cuarto del siglo pasado ya había sido introducido accidentalmente en Asia y Europa (Millikin \& Williams, 1984), donde continúa expandiéndose (Türeli et al. 2016; Pla Ventura et al. 2018). En Venezuela $C$. sapidus habita en toda la línea costera, y su distribución está muy influenciada por la temperatura; no reside en aguas que no superan los $20^{\circ} \mathrm{C}$ (Valentine et al. 1972).

C. sapidus tiene importancia económica en América, especialmente en México (Soto, 1980; Ramírez \& Hernández, 1988) y en Estados Unidos, el cual es considerado como el primer productor mundial (Williams, 1974; Villasmil \& Mendoza, 2001); pescándose, en ese país, la especie, también con fines recreacionales (Vincent et al. 2001; Atar \& Ceçer, 2003). Este valor se debe a que tiene talla grande y su carne es cotizada por el buen sabor; con un alto nivel de proteína (aproximadamente 16\%) y apenas $1 \%$ de grasa (Sañudo et al. 1997).

Según Casler \& García Pinto (2011a), pescadores de Barranquitas, caño la $\mathrm{O}$, Potreritos y puerto Concha, del estado Zulia, dependen casi exclusivamente de estos cangrejos para su subsistencia; al igual que en la Ceiba, estado Trujillo y Palmarito, estado Mérida (Perdomo et al. 2010). El objetivo del presente trabajo fue evaluar el efecto del manejo de la pesquería del cangrejo azul sobre la sostenibilidad del recurso en el lago de Maracaibo y aportar las bases para promover la adopción de medidas correctivas que garanticen su conservación.

\section{MATERIALES Y MÉTODOS}

El trabajo se realizó entre los años 2015 y 2018, como parte de un proyecto de investigación financiado parcialmente por la Universidad Experimental Sur del Lago (UNESUR), ubicada cerca de puerto Concha, Venezuela (Fig. 1). 


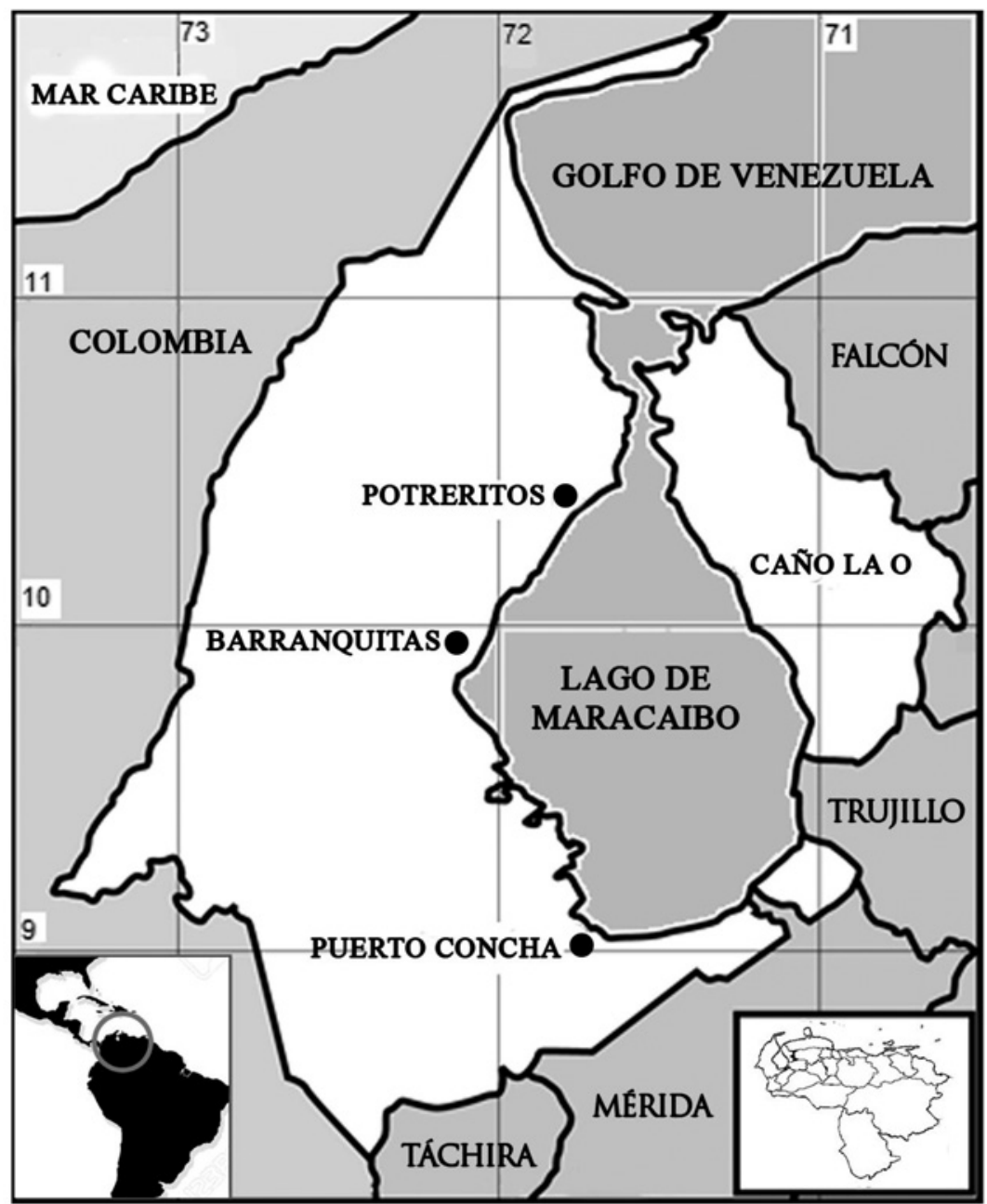

Fig. 1. Ubicación relativa nacional y regional del lago de Maracaibo. Se resaltan los puertos pesqueros más importantes. El área en blanco se corresponde con el estado Zulia

Fig. 1. Relative national and regional location of Lake Maracaibo. The most important fishing ports are highlighted. The white area corresponds to the Zulia State

La metodología consistió en el cultura (INSOPESCA) en los puertos análisis de los volúmenes históricos pesqueros del estado Zulia entre los de captura (tm/año) recopilados por el años 1984 y 2002, año a partir del cual Instituto Socialista de Pesca y Acui- hay un vacío de información; así como 
en la revisión de los estudios científicos realizados, algunos aún no publicados.

Específicamente se analizó la información publicada por Villasmil et al. (1996), sobre las estadísticas de captura y el esfuerzo de pesca, así como el trabajo de Ferrer (1997) acerca de la efectividad de dos tipos de nasas. Villasmil \& Mendoza (2001) describieron las características de las embarcaciones, artes y faenas de pesca, y utilizaron las estadísticas de las plantas procesadoras, y los muestreos semanales del personal del Instituto Nacional de Investigaciones Agropecuarias, para calcular el volumen de las capturas, la captura por unidad de esfuerzo y la cosecha máxima sostenible. Andrade de Pasquier et al. (2009) evaluaron el impacto de la pesca con palangre sobre la talla de los cangrejos, mientras que Perdomo et al. (2010) realizaron una evaluación de la pesca con palangre en el puerto la Ceiba, estado Trujillo; y Andrade de Pasquier et al. (2012) analizaron las relaciones biométricas y la composición por sexos de la especie.

Buonocore et al. (2011) describieron el ambiente abiótico de $C$. sapidus, mientras que Briceño et al. (2011) el ambiente biótico, Casler \& García Pinto (2011a) estudiaron la ecología, García Pinto et al. (2011a) la biología reproductiva y Andrade de Pasquier et al. (2011) la biología pesquera. Casler et al. (2011a) investigaron la conservación y mane- jo, mientras que García Pinto et al. (2011b) y González et al. (2011) los aspectos socioeconómicos y Casler et al. (2011b) los aspectos taxonómicos.

La información recopilada en eventos de carácter técnico-científico también fue tomada en consideración; eventos en los cuales participaron, en su mayoría, los autores del trabajo, así como en más de una docena de faenas realizadas en aguas del sur del lago, específicamente cerca de puerto Concha.

El análisis de la pesquería de C. sapidus se enfocó en la evolución histórica de los volúmenes de captura disponibles hasta el año 2002, en función de los cambios en los métodos de pesca, la relación de sexos y la talla de los individuos; igualmente en el uso de algunos resultados sobre crecimiento y mortalidad total (Z), mortalidad natural (M) y mortalidad por pesca (F) (Villasmil et al. 1997). En el trabajo también fueron considerados algunos resultados de crecimiento y mortalidad, aún no publicados, estimados por investigadores de la Universidad Experimental Rafael María Baralt (UNERBM). Para evaluar la intensidad de la extracción se calculó la tasa de explotación E, a partir de los valores obtenidos para $\mathrm{F}$ y $\mathrm{Z}(\mathrm{E}=\mathrm{F} / \mathrm{Z})$; se asume que un valor de $E>0.5$ indica una sobrepesca del recurso (Gulland, 1971; Díaz et al. 2014).

En el texto, la talla se expresa como el ancho del caparazón (AC); definido como la mayor separación 
entre las octavas espinas o salientes laterales del exoesqueleto (Casler et al. 2011b). Según la normativa vigente, en Venezuela la talla mínima permitida es $\mathrm{AC} \geq 8 \mathrm{~cm}$.

\section{RESULTADOS Y DISCUSIÓN}

\section{Relación macho:hembra}

Ferrer (1997), usando nasas en el norte del lago, registró $89 \%$ machos y $11 \%$ hembras (una relación de 9:1). Andrade de Pasquier et al. (2012) analizaron mensualmente el sexo de 300 individuos seleccionados al azar en los desembarcos de Barranquitas, puerto Concha, y caño la $\mathrm{O}$, entre septiembre de 2005 y diciembre de 2009, y encontraron una composición sexual de 1:1 entre machos y hembras; aunque observaron diferencias estadísticamente significativas entre los sexos en los cangrejos de mayor edad $(P<0.0001)$, con los machos más abundantes. Una proporción sexual favorable a los machos (9:1) también fue señalada por Ferrer (1997), al usar nasas en el norte del estuario, y por Mora \& Semprum (2008), en el sur. Según Mora \& Semprum (2008), en esta zona sur, la composición sexual de los cangrejos azules desembarcados en puerto Concha fue de $69 \%$ para machos y $31 \%$ para hembras (relación de 3:1), lo que según los autores se debe a que en el sur del lago la salinidad es menor, pues allí desembocan importantes afluentes, y los ma- chos se adentran más que las hembras en aguas con menor salinidad.

En general, los juveniles de la especie procedentes de aguas de mayor salinidad, donde nacen, se desplazan a áreas de menor salinidad donde crecen, alcanzan la madurez sexual y se aparean (Prager et al. 1990; Hsueh et al. 1993) aprovechando las áreas de manglar con abundante vegetación; por esta razón, las partes sur, sureste y suroeste del lago son claves para la especie, dado que allí existen manglares de considerable extensión (Medina \& Barboza, 2003). Aparentemente, en estas zonas de crecimiento, maduración y apareamiento la proporción sexual de C. sapidus es de 1:1, excepto algunas ocasiones en que tiende a favorecer a los machos como en los cangrejos de tallas grandes (Andrade de Pasquier et al. 2012), y en las zonas de reproducción, donde la salinidad es menor (Mora \& Semprum, 2008); manteniéndose actualmente estas proporciones, aunque aparentemente no existen trabajos que lo verifiquen.

\section{Talla}

Andrade de Pasquier et al. (2009) realizaron muestreos mensuales en los desembarcos de puerto Concha, Santa María, Barranquitas y Caño la O durante el período 20012007 (excepto el año 2004) y, según sus resultados, el AC promedio de los cangrejos se redujo desde 9.36 $\mathrm{cm}$ hasta $8.60 \mathrm{~cm}$ para la pesca con 
nasas, y se mantuvo en $9.30 \mathrm{~cm}$ para la pesca con palangres, durante el periodo 2003-2007. Esos resultados son similares a los encontrados por investigadores de la UNERMB para el año 2008, aunque no han sido publicados, en cuanto a un $\mathrm{AC}$ promedio para puerto Concha, Barranquitas y Caño la $\mathrm{O}$, de $9.1,9.0$ y $8.7 \mathrm{~cm}$, respectivamente. Andrade de Pasquier et al. (2009) encontraron que para el periodo $2001-2007,1.26 \%$ y $13.49 \%$ de los cangrejos pescados con nasas y palangres, en forma proporcional, exhibieron una talla menor a la permitida legalmente $(\mathrm{AC} \geq 8 \mathrm{~cm}$ ) en el país; siendo ello más evidente en el segundo caso, al encontrarse hasta $24.82 \%$ de los individuos con AC $<8 \mathrm{~cm}$ para el año 2007. Por su parte Mora \& Semprum (2008), encontraron un $9.81 \%$ y $12.45 \%$ de machos y hembras, desembarcados en puerto Concha con un AC $<8 \mathrm{~cm}$. Posteriormente, Andrade de Pasquier et al. (2012) determinaron un incremento $(16.80 \%)$ de cangrejos desembarcados con AC menor al establecido en la normativa vigente.

Aparentemente, los cangrejos cuyo tamaño está por debajo de la talla mínima permitida $(\mathrm{AC} \geq 8 \mathrm{~cm})$ que se están capturando en el lago representan un porcentaje más bien bajo $(12.0 \%$ en promedio), aun cuando el palangre utilizado durante los últimos años como equipo de pesca resulta ser menos selectivo en comparación con las nasas
(Andrade de Pasquier et al. 2009). Sin embargo, el promedio de las tallas desembarcadas $(\mathrm{AC}=8.9 \pm 0.3 \mathrm{~cm})$, aunque cumple con lo establecido en cuanto a la talla mínima, sí constituye un problema mayor por estar apenas por encima del valor permitido, y su captura podría estar afectando de forma significativa a la población. De allí que una medida inmediata para la conservación de este recurso es incrementar la talla mínima de captura; considerando que la vigente está por debajo de los valores señalados para las pesquerías de otras regiones como las de las costas de México; lo que incluye a Tamaulipas $(14.0 \mathrm{~cm})$, Veracruz $(15.0 \mathrm{~cm})$ y el golfo de California $(12.0 \mathrm{~cm})$ (Rosas-Correa y De Jesús-Navarrete, 2008). Sin embargo, para establecer un tamaño mínimo de captura más adecuado en el lago de Maracaibo es necesario tomar en cuenta de manera exacta la talla del cangrejo a la primera madurez; al considerar que actualmente la captura de individuos inmaduros sexualmente podría estar reduciendo el efecto de la veda y desvirtuando el esfuerzo global para la conservación y uso sostenible de la especie, la cual ha sido clasificada por los expertos como una especie vulnerable de extinción y, por lo tanto, su captura en etapas inmaduras constituye una amenaza para la renovación de las poblaciones del recurso pesquero de mayor importancia socioeconómica en el lago (Casler \& García Pinto, 2011a). 
Peso total de las capturas y su relación con el cambio de métodos de pesca

Hasta el año 2001 en el lago de Maracaibo C. sapidus se pescaba con nasa, un dispositivo que consiste en una jaula, que según las normas vigentes, debe tener como máximo $45 \mathrm{~cm}$ (largo $\mathrm{x}$ alto $\mathrm{x}$ ancho), estar recubierta de una rejilla de metal o plástico y tener un orificio de por lo menos $5 \mathrm{~cm}$ de ancho en sus lados frontal y posterior, y de $2.5 \mathrm{~cm}$ en el resto; el orificio de entrada debe ser ovalado u oblongo y debe medir $16 \mathrm{~cm}$ de ancho y de 6 a $8.6 \mathrm{~cm}$ de alto (Instituto Socialista de la Pesca y Acuicultura, 2010). Detalles sobre este equipo pueden verse en Andrade de Pasquier et al. (2009).

A partir del año 2002 se comenzó a pescar C. sapidus con palangre (An- drade de Pasquier et al. 2009; Perdomo et al. 2010); el cual está construido con una línea de polietileno de longitud variable, con plomadas a intervalos de 1 m que arrastran la línea hacia el fondo, y cientos de brazoladas amarradas a la línea principal, entre las plomadas. En el extremo de cada brazolada se coloca un amarre denominado "gaza" en el que se sujeta una cabeza de pollo ( $G a$ llus domesticus) que sirve de carnada (Villasmil \& Mendoza, 2001; Perdomo, 2010, Fig. 2). La adopción del palangre para la captura del cangrejo azul fue debido a que los costos de construcción y mantenimiento de las nasas eran y siguen siendo insostenibles. El palangre es un equipo más versátil, pues no solo es menos costoso, sino que ocupa poco espacio en las embarcaciones (Andrade de Pasquier et al. 2011).
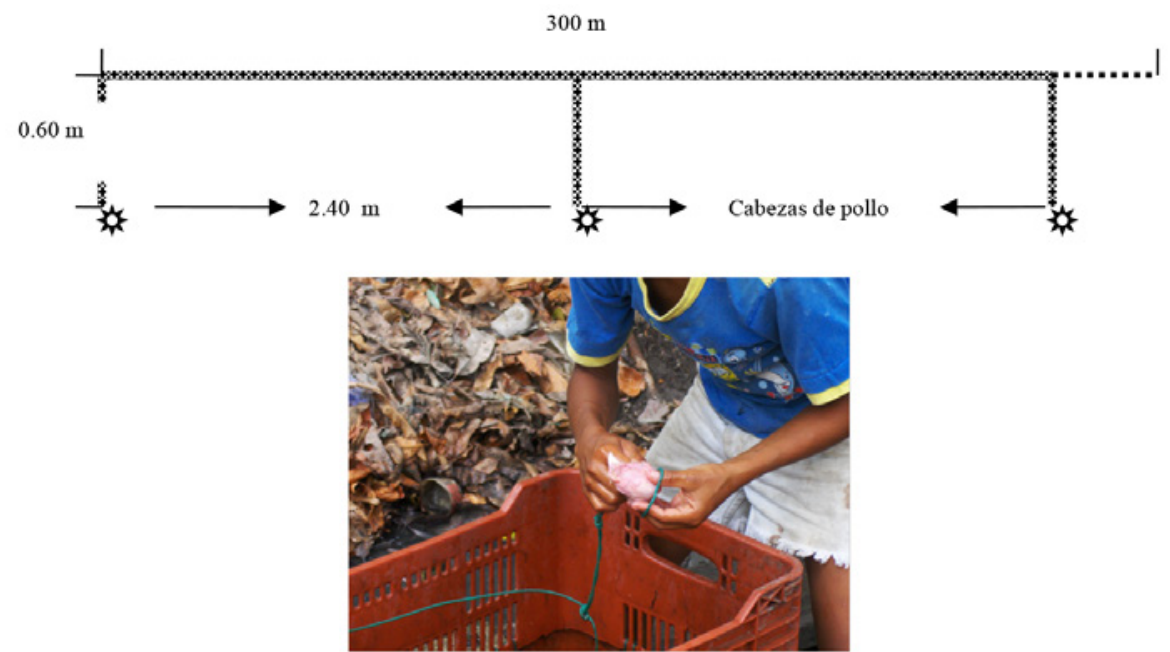

Fig. 2. Dibujo de un palangre típico (arriba). Cesta en la que se transporta y amarre de la carnada (abajo)

Fig. 2. Drawing of a typical longline (above). Basket in which it is transported and bait tie (down) 
Desde la implementación del palangre ha sido evidente el aumento del éxito en la captura (Fig. 3), duplicándose, por ejemplo, desde 7 mil tm durante el período 1998-2002 hasta 14.3 mil tm durante el período 2003-2009 (INSOPESCA; Andrade de Pasquier et al. 2011). La selectividad del palangre, sin embargo, se redujo, en comparación con la nasa al permitir la captura de una mayor cantidad de individuos con tallas inferiores a la mínima legalmente permitida; hasta el punto de que el porcentaje de estos individuos aumentó desde $1.44 \%$ en 2003 hasta $24.82 \%$ en 2007 (Andrade de Pasquier et al., 2009) y actualmente está alrededor del 50\%, según comunicación personal. Este hecho se agrava debido a la rapidez con que se realiza la recolección de los palangres en varias áreas del lago, para procurar un mayor número de cangrejos; no siendo posible hacer la selección in situ de los individuos según su talla, ni regresar al lago a aquellos cuyas tallas son inferiores a la reglamentaria; lo que, posiblemente, está reduciendo el stock de pesca (Mora \& Semprum (2008). Rugolo (1998) advierte que el riesgo de colapso de una pesquería es elevado cuando el esfuerzo se mantiene alto, mientras se reduce el tamaño de la población.

La pesquería del cangrejo azul en el lago de Maracaibo es clasificada como de tipo artesanal (Villasmil et al. 1996; Ferrer, 1997; Mora \& Semprum, 2008; Andrade de Pasquier et al. 2011),

\section{Peso de las capturas (1984-2002)}

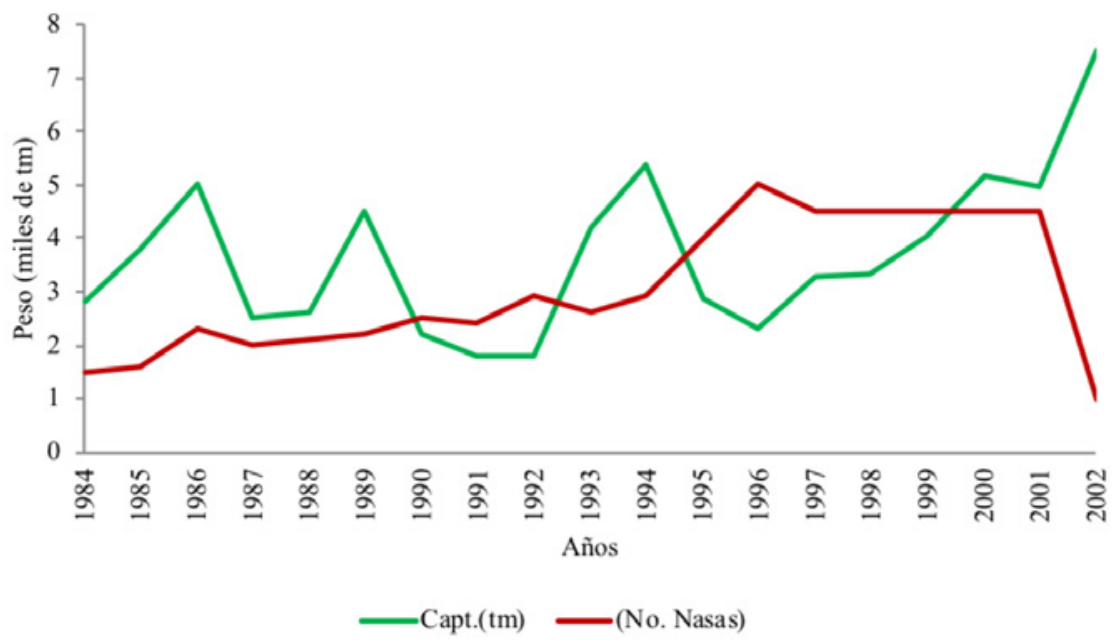

Fig. 3. Peso de las capturas de cangrejos azules en el lago de Maracaibo entre los años 1984 y 2002. No hay datos disponibles a partir del año 2002

Fig. 3. Weight of blue crab caught in Lake Maracaibo between 1984 and 2002. No data is available after 2002 
sin embargo, su captura se realiza a través de técnicas propias de la pesca comercial, al considerar que la cantidad de pescadores y dispositivos de pesca, y por ende, la cantidad de cangrejos pescados anualmente, no se corresponde con la definición de la FAO (2019). Un ejemplo de pesquería artesanal es el de la bahía de Chetumal en México, que tiene solamente de 4 a 5 pescadores constantes y de 15 a 40 trampas/pescador (Rosas-Correa y De Jesús-Navarrete, 2008).

\section{Crecimiento y mortalidad}

La longitud asintótica $\left(L_{\infty}\right)$ de C. sapidus en el lago de Maracaibo es de $14.85 \mathrm{~cm}$ (Villasmil y Mendoza,
1997), y es inferior al reportado por Rosas-Correa y De Jesús-Navarrete (2008) para la bahía de Chetumal, por Rugolo et al. (1998) para la bahía de Chesapeake, por Hesler et al. (2001) para Delaware, y por el Instituto Nacional de Pesca de México (2000) para el golfo de México (Cuadro 1); lógicamente con un valor de la tasa de crecimiento $(K)$ más alto $\left(1.65 \mathrm{año}^{-1}\right)$, pues toma en cuenta que si $\mathrm{K}$ se refiere a la rapidez con que el cangrejo alcanza el $L_{\infty}$ (Gulland, 1971), esta longitud asintótica será alcanzada más rápido en el lago de Maracaibo por ser más pequeña.

Cuadro 1. Valores de los parámetros de crecimiento, mortalidad y tasa de explotación para C. sapidus en el lago de Maracaibo en comparación con los de otras pesquerías

Table 1. Values of growth, mortality, and exploitation rate parameters for $C$. sapidus in Lake Maracaibo compared to those of other fisheries

\begin{tabular}{lcccccc}
\hline Parámetro & $\begin{array}{l}\text { Bahía de Chetumal } \\
\text { Rosas-Correa y De } \\
\text { Jesús-Navarrete } \\
(2008)\end{array}$ & $\begin{array}{l}\text { Chesapeake } \\
\text { Rugolo } \text { et al. } \\
(1998)\end{array}$ & $\begin{array}{l}\text { Delaware } \\
\text { Hesler } \text { et al. } \\
(2001)\end{array}$ & $\begin{array}{l}\text { Golfo de } \\
\text { México } \\
\text { INP (2000) }\end{array}$ & $\begin{array}{l}\text { Lago de } \\
\text { Maracaibo } \\
\text { Villasmil \& } \\
\text { Mendoza (1997) }\end{array}$ & $\begin{array}{l}\text { Lago de } \\
\text { Maracaibo } \\
\text { UNERMB, datos } \\
\text { no publicados. }\end{array}$ \\
\hline $\begin{array}{l}\text { Crecimiento } \\
\mathrm{L}_{\infty}\end{array}$ & 23.15 & 26.25 & 23.47 & 20.20 & 14.85 & 16.80 \\
$\mathrm{~K}\left(\mathrm{añno}^{-1}\right)$ & 0.51 & 0.59 & 0.75 & 0.75 & 0.17 & 0.84 \\
Mortalidad & 0.85 & 1.55 & 1.17 & 3.91 & 0.95 & 7.53 \\
$\mathrm{Z}$ & 0.66 & 0.37 & 0.75 & 1.59 & 0.25 & 1.87 \\
$\mathrm{M}$ & 0.19 & 1.18 & 0.42 & 2.32 & 0.70 & 5.66 \\
$\mathrm{~F}$ & & & & & & \\
Tasa de & 0.22 & 0.75 & 0.36 & 0.59 & 0.74 & 0.75 \\
Explotación $\mathrm{E}$ & & & & & & \\
\hline
\end{tabular}


La mortalidad total $(Z)$ para la época en que $C$. sapidus era capturado con nasas fue estimada en $9.5 \mathrm{año}^{-1}$, la mortalidad natural (M) en 2.5 año $^{-1}$ y la mortalidad por pesca $(\mathrm{F})$ en $7.0 \mathrm{año}^{-1}$ (Cuadro 1); observándose que ya para esa época la mortalidad total $(Z)$ era relativamente alta y estaba asociada a una alta tasa de mortalidad por pesca (F), como consecuencia de una muy alta intensidad de extracción (Villasmil \& Mendoza, 1997). Para aquella época la tasa de explotación $E$ era de 0.74 y estaba por encima del valor límite de 0.5 que indica una sobrepesca (Gulland, 1971; Díaz et al. 2014); un nivel de explotación que aparentemente se ha mantenido durante los últimos años con el uso del palangre como dispositivo principal en las capturas, por cuanto según estimaciones de investigadores de la UNERMB, aún no publicados, la tasa de explotación (E) actualmente es de 0.75 (Cuadro 1).

Durante el período 1984-1997 el esfuerzo de pesca se incrementó de manera constante, lo que ocasionó una disminución notable de la CPUE y de la biomasa (Villasmil \& Mendoza, 2001); por lo que, posiblemente, la mortalidad por pesca haya excedido el umbral que reduce la biomasa del stock reproductor al 10\% del stock virgen propuesto por Rugolo et al. (1998) y por Miller \& Houde (1998) y que según Sharov et al. (2003) indica sobrepesca.

\section{Aspectos ecológicos y ambientales}

El cangrejo azul es un depredador de algunas ostras (Eggleston, 1990) y almejas (Blundon \& Kennedy, 1982; Sponaugle \& Lawton, 1990; Smith \& Haines, 1991; Eggleston et al. 1992), entre las que cuentan algunas especies de importancia comercial (Vincent et al. 2001); siendo este rol ecológico el determinante biótico más importante de la estructura de la comunidad (Bromilow, 2017), la cual actualmente debe verse afectada por la disminución de la biomasa del cangrejo azul, aunque aún no hay estudios que lo demuestren. Por otro lado, las megalopas de este crustáceo son depredadas por algunos peces (Millikin \& Williams, 1984; Türeli et al. 2016; Bromilow, 2017), así como cangrejos juveniles y adultos (Fox \& White, 1969); que también son depredados por caimanes, tortugas, aves y mamíferos, principalmente zorros cangrejeros (Valentine $e t$ al. 1972), así como por perros de agua (Shanks, 2002) y nutrias (Chabreck et al. 1982.

La introducción de pavones $(\mathrm{Ci}$ chla orinocensis y C. temensis) desde 1965, su reciente multiplicación en las lagunas de Ologá y Congo Mirador, cerca de la desembocadura del río Catatumbo, y su dispersión hacia el norte en la laguna Manatí, son hechos significativos desde el punto de vista ecológico (Lasso-Alcalá et al. 2014) que pueden estar alterando las poblaciones 
de invertebrados acuáticos inclusive la de los cangrejos azules, tal como ha ocurrido en Brasil (Pinto-Coelho et al. 2008); al considerar que los pavones son peces carnívoros y territoriales, y además muestran una baja mortalidad natural.

La contaminación del lago por hidrocarburos, posiblemente está afectando también la población del cangrejo azul, como lo han señalado muchos actores, incluso los pescadores, al denunciar una red de oleoductos donde con frecuencia ocurren fugas de petróleo y derivados que causan daños visibles en el hábitat de la fauna acuática, lo que reduce su idoneidad; fugas que al entrar en contacto con los cangrejos provocan su evasión y reducción del forrajeo, forza una disminución de su presencia en el área contaminada (Culberston et al. 2007), y en consecuencia, afecta negativamente la continuidad de la pesquería.

Pero la contaminación generada por otras fuentes al sur del lago, donde la agricultura y ganadería son muy dinámicas, puede tener un impacto negativo de mayor magnitud aun; producto del uso excesivo de insecticidas y fungicidas, entre otros agroquímicos, y la eutrofización por la descarga de fertilizantes químicos y excretas de humanos y animales domésticos, a los cuerpos de agua tributarios, por lo general sin pasar por ningún sistema de tratamiento (Molina, 2009).
Rivas et al. (2009) evaluaron el aporte de N y P de los ríos Limón, Palmar, Santa Ana, Catatumbo, Escalante, Chama, Motatán, Misoa, Machango y Pueblo Viejo, que en conjunto agregan más de tres cuartas partes del agua dulce que ingresa al lago, y encontraron valores promedio de 1.20 y $0.84 \mathrm{mg} \mathrm{l}^{-1}$, respectivamente, que contribuyen a su eutrofización. Este proceso favorece la proliferación de algas como la lenteja de agua (Lemna sp.), que ha llegado a cubrir hasta $16 \%$ del espejo de agua del lago, que reduce la tasa fotosintética del plancton y, en consecuencia, disminuye el aporte de oxígeno; perjudicando de esta manera a toda la biota (Aldana et al. 2006) y por ende a la población del cangrejo azul. La descomposición de la materia orgánica que aporta la Lemna sp. muerta es realizada por bacterias que compiten por oxígeno, lo que incrementa su demanda biológica y acentúa la anoxia del lago, y a la vez, afecta negativamente la calidad microbiológica de los tejidos del cangrejo que se comercializa (Morillo et al. 2007).

\section{Aspectos de naturaleza sociocultu- ral y sociopolítica}

C. sapidus, es una especie comercialmente muy importante en el lago y actualmente cuenta entre los recursos más explotados por los pescadores artesanales; compitiendo en el nivel de producción con especies de camarones peneidos y con 
peces como la curvina (Cynoscion acoupa), lisas (Mugil sp.) y bocachico (Prochüodus reticulatus). La pesquería de $C$. sapidus, además de beneficiar directamente a las poblaciones adyacentes al lago, constituye una fuente de divisas para el país, dado que más del 99\% del producto final procesado por la industria de carne de cangrejo se destina a la exportación (Villasmil y Mendoza, 1997).

A pesar de su importancia comercial, aparentemente, no existen medidas adecuadas para la conservación del recurso y muchas han sido establecidas por consulta a los pescadores, que, si bien conocen aspectos generales del recurso, el conocimiento que tienen sobre los aspectos biológicos y ecológicos de la especie es escaso. Los detalles técnicos sobre el manejo y la guardería del recurso deben ser discutidos entre los expertos y los representantes del Estado, y las medidas adoptadas deben ser informadas a los pescadores, quienes tienen la obligación de aceptar las disposiciones que se establezcan y no reaccionar negativamente a las resoluciones como generalmente ocurre. Mora \& Semprum (2008) señalan, por ejemplo, que los pescadores de puerto Concha por lo general no son supervisados in situ por los inspectores; evidenciándose una falla grave que pudiera estar ocurriendo de manera generalizada en el lago.

\section{Aspectos legales}

La resolución N. ${ }^{\circ} 052$ del 5 de agosto de 2010 sobre la veda del cangrejo azul en el lago de Maracaibo (Instituto Socialista de la Pesca y Acuicultura, 2010), que abarca desde el 15 de agosto hasta el 30 de septiembre, permite solo un palangre de hasta $400 \mathrm{~m}$ por embarcación. Permite su pesca entre las 5:00 a. m. y las 6:00 p. m., excepto desde el norte del puente sobre el lago hasta la bahía El Tablazo, prohíben la extracción de las hembras ovadas, independientemente de su talla. Esta resolución se cumple parcialmente: se respeta la veda, pero no la longitud de los palangres que en algunos casos supera los $1000 \mathrm{~m}$.

La Ley Orgánica de Seguridad y Soberanía Agroalimentaria (Gaceta Oficial de la República Bolivariana de Venezuela, 2008b), puntualiza la responsabilidad que tiene el Estado para garantizar la disponibilidad, acceso, intercambio y distribución equitativa de los alimentos. Esta ley, además, prioriza la importancia de las comunidades de pescadores artesanales en la búsqueda de la seguridad agroalimentaria.

La continuidad del cangrejo azul como recurso biológico no está siendo garantizada debido a que depende del éxito de captura más que de una regulación a través de medidas proteccionistas (Mendoça et al. 2010) como las que se estipulan en el artículo 2 de la Ley de Gestión de la Diversidad Biológica (Gaceta Oficial de la República 
Bolivariana de Venezuela, 2008a) y en la Ley de Pesca y Acuicultura (Gaceta Oficial de la República Bolivariana de Venezuela, 2009). Es evidente que esta ley tampoco se está cumpliendo a cabalidad y, ante la realidad descrita, lo prioritario debe ser, precisamente, regular la pesca.

Es incuestionable que no se está asegurando la renovación natural del recurso para garantizar la producción y cubrir la demanda del mercado porque, aparte de la contaminación del Lago, hay evidencias de que se está sobreexplotando. Lamentablemente hasta el presente no se les ha otorgado la debida importancia a las estadísticas de pesca ni a los aspectos biológicos y técnicos que vienen siendo advertidos por los investigadores. Por otra parte, la protección de los pescadores artesanales y sus caladeros de pesca no se está llevando a la práctica (Molina et al. 2013).

La Ley de Zonas Especiales de Desarrollo Sustentable (Gaceta Oficial de la República Bolivariana de Venezuela, 2001) tiene entre sus objetivos ejecutar planes y fomentar una explotación de los recursos de manera que se pueda elevar el bienestar social y la calidad de vida de la población. El uso insostenible del que está siendo objeto el cangrejo azul en el lago de Maracaibo deja en evidencia que esta ley tampoco se está cumpliendo.

\section{CONCLUSIONES}

El cambio de arte de pesca de nasas a palangres determinó un aumento significativo en el éxito de capturas del cangrejo azul y una reducción de la selectividad por talla, lo que no ha tenido una respuesta administrativa efectiva y a tiempo por parte del Estado venezolano.

Por otra parte, las investigaciones reflejan una reducción sostenida del AC y extracción a una tasa creciente de individuos sexualmente inmaduros.

La longitud asintótica $\left(L_{\infty}\right)$ alcanzada es inferior al reportado para la bahía de Chesapeake, bahía de Delaware, golfo de México y bahía de Chetumal, en México; con una mortalidad total $(Z)$ y por pesca $(F)$ significativamente alta, que determinan una tasa de explotación (E) por encima del valor límite de 0.5 y que indica una sobreexplotación del recurso.

Los pescadores no respetan a cabalidad la legislación vigente y el Estado ha fallado en el registro de datos, en la evaluación del hábitat y en la gestión del recurso, tomando, en algunos casos, decisiones más políticas que técnicas.

Para garantizar el uso sostenible de la especie en el lago de Maracaibo es imperativo incrementar la talla mínima de primera captura legalmente permitida $(\mathrm{AC} \geq 8.0 \mathrm{~cm})$, como ocurre en otras regiones como la costa de 
México, hacer cumplir a cabalidad las regulaciones vigentes, y las que se establezcan, así como diseñar e implementar un plan de manejo que incluso beneficiaría también al resto de los recursos naturales del sistema lacustre.

\section{AGRADECIMIENTOS}

A los pescadores de puerto Concha por su apoyo logístico durante el trabajo de campo. A la Universidad Experimental Sur del Lago por su apoyo financiero. A los tres revisores anónimos quienes examinaron el manuscrito y realizaron aportes importantes para la finalización de esta publicación. Al equipo editorial y evaluador de la Revista Ciencias Marinas y Costeras; sin su paciencia y dedicación no habría sido posible terminar el trabajo.

\section{BIBLIOGRAFÍA}

Aldana, G. Troncone, F., Ochoa, E., Márquez, R. \& Rivas, Z. (2006). Posible explicación al fenómeno de crecimiento masivo de Lemna obscura (Lemnaceae) en el Lago de Maracaibo (Estado Zulia, Venezuela). Ciencia, 14(2), 7-21.

Andrade de Pasquier, G., Ramírez, S., García, P. L., Buonocore, R. \& Delgado, J. (2009). Impacto del palangre para la captura del cangrejo azul Callinectes sapidus en la composición por tallas de los desembarques en el Lago de Maracaibo, Venezuela. Proceedings of the 62 nd Gulf and Caribbean Fisheries. Cumaná, Venezuela.
Andrade de Pasquier, G., Ferrer, O. \& Buonocore, R. (2011). Biología Pesquera. En C. Casler, L. García-Pinto \& C. Sangronis, (Eds.), El cangrejo azul en el sistema del lago de Maracaibo. Ecología, pesquería y aspectos socioeconómicos (pp. 123-156). Maracaibo, Venezuela: Ediciones Astro Data S. A. Andrade de Pasquier, G., García P., L., Buonocore, R. \& Méndez, Y. (2012). Relaciones biométricas y proporción de sexos del cangrejo azul, Callinectes sapidus (Rathbun, 1896), en el lago de Maracaibo, Venezuela. Ciencia, 20(3), 155-158.

Atar, H. \& Ceçer, S. (2003). Width/length-weight relationships of the blue crab (Callinectes sapidus Rathbun 1896) Population living in Meymelek Lagoon Lake. Turk. J. Fish. Aq. Sci., 27, 443447.

Blundon, J. A. \& Kennedy, V. S. (1982). Mechanical and behavioural aspects of the blue crab, Callinectes sapidus Rathbun predation on Chesapeake Bay bivalves. J. Exper. Mar. Biol. Ecol., 65, 47-65. https://doi.org/10.1016/00220981(82)90175-7.

Briceño, H., Buonocore, R., Sangronis, C., \& Rojas, J. (2011). Ambiente biótico. En C. Casler, L. GarcíaPinto \& C. Sangronis, (Eds.), El cangrejo azul en el sistema del lago de Maracaibo. Ecología, pesquería y aspectos socioeconómicos (pp. 6182). Maracaibo, Venezuela: Ediciones Astro Data S. A.

Bromilow, A. M. (2017). Juvenile blue crab survival in nursery habitats: predator identification and predation impacts in Chesapeake Bay. Master Thesis not published. School of Marine Science, College of William and Mary in Virginia. 
Buonocore, R., Briceño, H. \& Sangronis, C. (2011). Ecología. En C. Casler, L. García-Pinto \& C. Sangronis, (Eds.), El cangrejo azul en el sistema del lago de Maracaibo. Ecología, pesquería y aspectos socioeconómicos (pp. 35-60). Maracaibo, Venezuela: Ediciones Astro Data S. A.

Casler, C. \& García Pinto, L. (2011a). Introducción. En C. Casler, L. García-Pinto \& C. Sangronis, (Eds.), El cangrejo azul en el sistema del lago de Maracaibo. Ecología, pesquería y aspectos socioeconómicos (pp. 17-30). Maracaibo, Venezuela: Ediciones Astro Data S. A.

Casler, C. \& Garcia Pinto, L. (2011b). Ecología. En C. Casler, L. García-Pinto \& C. Sangronis, (Eds.), El cangrejo azul en el sistema del lago de Maracaibo. Ecología, pesquería y aspectos socioeconómicos (pp. 83-100). Maracaibo, Venezuela: Ediciones Astro Data S. A.

Casler, C., García Pinto, L., Buonocore, R., \& Andrade de Pasquier, G. (2011a). Conservación y manejo. En C. Casler, L. García-Pinto \& C. Sangronis, (Eds.), El cangrejo azul en el sistema del lago de Maracaibo. Ecología, pesquería y aspectos socioeconómicos (pp. 157184). Maracaibo, Venezuela: Ediciones Astro Data S. A.

Casler, C., García Pinto, L., \& Sangronis, C. (2011b). Identificación de los portúnidos. En C. Casler, L. García-Pinto \& C. Sangronis, (Eds.), El cangrejo azul en el sistema del lago de Maracaibo. Ecología, pesquería y aspectos socioeconómicos (pp. 231-262). Maracaibo, Venezuela: Ediciones Astro Data S. A.

Chabreck, R. H., Holcombe, J. E., Liscombe, R. G. \& Kinler, N. E. (1982). Winter foods of river otters from saline and fresh environments in Louisiana. Proc. Ann. Conf. South. Assoc. Fish Wild. Ag., 36, 473-484.
Culberston, J., Valiela, I., Peacock, E., Reddy, C. M., Carter, A. \& VanderKruik, R. (2007). Long-term biological effects of petroleum residues on fidden crabs in salt marshes. Mar. Poll. Bull., 54, 955962. https://doi.org/10.1016/j.marpolbul.2007.02.015.

Díaz. A., Ferrer, O., Álvarez, R., González, L., Méndez, J., \& Corona, M. (2014). Mortality, recruitment pattern and growth of the White shrimp (Litopenaeus schmitti (Crustacea: Penaeidae) from the Gulf of Venezuela. Ciencia, 22(4), 187-196.

Eggleston, D. B. (1990). Behavioural mechanisms underlying variable functional responses of blue crabs, Callinectes sapidus feeding on juvenile oysters, Crassostrea virginica. J. Anim. Ecol., 59(2), 615-630. https://doi.org/10.2307/4884.

Eggleston, D. B, Lipcius, R. N. \& Hines, A. H. (1992). Variation in density-dependent predation by blue crabs upon infaunal clam species with contrasting distribution and abundance patterns. Mar. Ecol. Prog. Ser., 85, 55-68. https://doi. org/10.3354/meps085055.

FAO. (2019). Fisheries and Aquaculture topics. Fisheries technology. Recuperado en abril 25, 2019, disponible en http://www.fao.org/fishery/

Ferrer, M. O. J. (1997). Effectiveness of two pots and others factors for harvesting hard blue crabs Callinectes sapidus in Lake Maracaibo, Venezuela. Ciencia, 5(2), 111-118.

Fox, L. S. \& White, C. J. (1969). Feeding habits of the southern flounder, Paralichthys lethostigma, in Barataria Bay, Louisiana. Proc. Louis. Acad. Sci., 32, 31-38.

Gaceta Oficial de la República Bolivariana de Venezuela. (2001). Ley de Zonas Especiales de Desarrollo Sustentable. Gaceta N. ${ }^{\circ}$ 5556, 13 de noviembre de 
2001. Caracas, Venezuela, Servicio Autónomo Imprenta Nacional y Gaceta Oficial.

Gaceta Oficial de la República Bolivariana de Venezuela. (2008a). Ley de Gestión de la Diversidad Biológica. Gaceta N. ${ }^{\circ}$ 39070, 01 de diciembre de 2008. Caracas, Venezuela, Servicio Autónomo Imprenta Nacional y Gaceta Oficial.

Gaceta Oficial de la República Bolivariana de Venezuela. (2008b). Ley de Seguridad y Soberanía Agroalimentaria. Gaceta N. ${ }^{\circ}$ 5889, 31 de julio de 2008. Caracas, Venezuela, Servicio Autónomo Imprenta Nacional y Gaceta Oficial.

Gaceta Oficial de la República Bolivariana de Venezuela. (2009). Decreto con Fuerza de Ley de Pesca y Acuicultura. Gaceta N. ${ }^{\circ}$ 5877, 11 de marzo de 2009. Caracas, Venezuela, Servicio Autónomo Imprenta Nacional y Gaceta Oficial.

García Pinto, L., Sangronis, C., Buonocore, R., \& Briceno, H. (2011a). Biología reproductiva. En C. Casler, L. García-Pinto \& C. Sangronis, (Eds.), El cangrejo azul en el sistema del lago de Maracaibo. Ecología, pesquería y aspectos socioeconómicos (pp. 103-122). Maracaibo, Venezuela: Ediciones Astro Data S. A.

García Pinto, L., Buonocore, R., Sangronis, C., \& Chirinos, J. (2011b). Procesamiento de la carne. En C. Casler, L. García-Pinto \& C. Sangronis, (Eds.), El cangrejo azul en el sistema del lago de Maracaibo. Ecología, pesquería y aspectos socioeconómicos (pp. 187204). Maracaibo, Venezuela: Ediciones Astro Data S. A.

González, A., Ferrer, O., \& Villarreal, A. (2011). Diagnóstico socioeconómico. En C. Casler, L. García-Pinto \& C. Sangronis, (Eds.), El cangrejo azul en el sistema del lago de Maracaibo. Ecología, pesquería y aspectos socioeconómicos (pp. 205-228). Maracaibo, Venezuela: Ediciones Astro Data S. A.

Gulland, J. A. (1971). Manual de métodos para la evaluación de poblaciones de peces. Zaragoza, España. Editorial Acribia.

Helser, T. E. \& Khan, D. M. (2001). Stock Assessment of Delaware Bay blue crab (Callinectes sapidus) for 2001. Delaware, EE. UU.: Division of Fish and Wildlife.

Hines, A. H., Lipcius, R. N. \& Haddon, M. (1987). Population dynamics and habitat partitioning by size, sex, and molt stage of blue crabs Callinectes sapi$d u s$ in a sub-estuary of central Chesapeake Bay. Mar. Ecol. Prog. Ser., 36, 55-64.

Hsueh, P. W., Mcclintock, J. B. \& Hopkins, T. S. (1993). Population dynamics and life history characteristics of the blue crabs Callinectes similaris and C. sapidus in Bay environments of the northern Gulf of Mexico. Mar. Ecol., 14(3), 239-257.

Instituto Nacional de la Pesca. (2000). La pesquería de jaiba del golfo de México y mar Caribe. México D. F. Diario Oficial de los Estados Unidos Mexicanos.

Instituto Socialista de la Pesca y Acuicultura. (2010). Normas técnicas de ordenamiento para regular la pesquería artesanal del cangrejo azul, Callinectes sapidus, en el Lago de Maracaibo y el Golfo de Venezuela. Resolución N. $^{\mathrm{o}}$ 052, 05 de agosto de 2010. Caracas, República Bolivariana de Venezuela. Servicio Autónomo Imprenta Nacional y Gaceta Oficial.

Lasso-Alcalá, O., Andrade de Pasquier, G., Hoyos, C., Bottini, B. \& Hernández, M. (2014). Sobre la introducción de los pavones Cichla orinocensis y $C$. temesis (Perciformes, Cichlidae), en la Cuenca del Lago de Maracaibo, Venezuela. Anartia, 26, 31-50. 
Mascialgiolli, P. \& Fébres, G. (2000). Climatología de la cuenca de Maracaibo. En G. Rodríguez, (Ed.), El sistema de Maracaibo (pp. 21-32). Caracas, Venezuela: Instituto Venezolano de Investigaciones Científicas (IVIC).

Medina, E. \& Barboza, F. (2003). Manglares del sistema del lago de Maracaibo: caracterización fisiográfica y ecológica. Ecotropicos, 16(2), 75-82.

Mendoça, J. T., Verani, J. R., \& Nordi, N. Evaluation and management of blue crab Callinectes sapidus (Rathbun 1896) (Decapoda - Portunidae) fishery in the Estuary of Canéia, Iguape and Ilha Comprida, Săo Paulo, Brazil. Braz. J. Biol., 70(1), 37-45.

Miller, T. J. \&. Houde, E. D. (1998). Blue crab target setting. Chesapeake, EE. UU.: Chesapeake Biological Laboratory.

Millikin, M. R. \& Williams, A. B. (1984). Synopsis of biological data on blue crab, Callinectes sapidus Rathbun. Washington, EE. UU.: Department of Commerce.

Molina, M. (2009). Fundamentos de factores naturales y producción agropecuaria. Santa Bárbara de Zulia, Venezuela: Consejo Editorial de la Universidad Nacional Experimental Sur del Lago.

Molina, M., Mejias, D., Torres, L., Arvelo, F. \& Yarumare, C. (2013). Aspectos soioeconómicos, administrativos, técnicos y legales de la pesquería del cangrejo azul (Callinectes sapidus) en Puerto Concha, sur del lago de Maracaibo. Agroalimentaria, 19(37), 109125.

Mora, A. \& Semprum, E. (2008). Evaluación de algunos aspectos pesqueros y socioeconómicos del Municipio Colón, Estado Zulia. Tesis no publicada, Universidad Nacional Experimental Sur del Lago, Santa Bárbara de Zulia, Venezuela.
Morillo, N., Rondón, I., Valero-Leal, K. \& Uzcátegui-Bracho, S. (2007). Bacterias Patógenas en carne de cangrejo comercializado fresco y pasteurizado. Maracaibo, Venezuela. Rev. Cient. FCV-LUZ, 17, 288-293.

Perdomo, D., García, D., González-Estopiñán, M., Santos, O., Moratinos, P., Cova, L. \& Medina, M. (2010). Evaluación de la pesca artesanal con palangre del cangrejo azul (Callinectes spp.), en el Puerto de La Ceiba, estado Trujillo, Venezuela. Zoot. Trop., 28(2), 221-237.

Pinto-Coelho, R., Bezerra-Neto, J., Miranda, F., Mota, T., Resck, R., Santos, A., Maia-Barbosa, P., Mello, N., Marques, M., Campos, M. \& Barbosa, F. (2008). The inverted trophic cascade in tropical plankton communities: Impacts of exotic fish in the Middle Rio Doce lake district, Minas Gerais, Brazil. Braz. J. Biol., 68(Suppl. 4), 1025-1037. http://dx.doi.org/10.1590/S151969842008000500010.

Pla Ventura, M., Quiñonero Salgado, S., Hernández Núñez, J., Velázquez Cano, J., Risueño Mata, P., \& López Soriano, J. (2018). Predation of the blue crab Callinectes sapidus Rathbun, 1896 on freshwater bivalves (Unionidae \& Corbiculidae) in eastern Iberian Peninsula. Folia Conch., 47, 3-9.

Prager, M. H., Mcconaugha, J. R., Jones, C. M. \& Geer, P. J. (1990). Fecundity of blue crab, Callinectes sapidus, in Chesapeake Bay: biological, statistical and management considerations. Bull. Mar. Sci., Univ. Miami, 46, 170-179.

Ramírez, M. S. \& Hernández, I. (1988). Investigación biologicopesquera para la obtención de la jaiba suave Callinectes spp en la Laguna de Alvarado, Veracruz. Tesis no publicada, Universidad Nacional Autónoma de México. 
Rathbun, M. S. (1986). The genus Callinectes. Proc. U.S. Nat. Mus., 18(1070), 349375.

Rivas, Z., Sánchez, J., Troncone, F., Márquez, R., Ledo de Medina, H., Colina, M. \& Gutiérrez, E. (2009). Nitrógeno y fósforo totales de los ríos tributarios al sistema Lago de Maracaibo, Venezuela. Interciencia, 34(5), 308-314.

Rosas-Correa, C. \& de Jesús-Navarrete, A. (2008). Parámetros poblacionales de la jaiba azul Callinectes sapidus (Rathbun, 1896) en la bahía de Chetumal, Quintana Roo, México. Rev. Biol. Mar. Ocean., 43(2), 247-253. http://dx.doi.org/10.4067/S071819572008000200003.

Rugolo L. J., Knotts, K. S., Lange, A. M. \& Crecco, V. A. (1998). Stock assessment of Chesapeake Bay blue crab (Callinectes sapidus Rathbun). J. Shellfish Res., 17, 906-930.

Sañudo, C., Del Mar, M. \& Beltrán, J. A. (1997). Calidad comercial de los productos de la acuicultura. En C. Buxadé (Ed.), Zootecnia, Bases de la Producción Tropical (pp. 349-369). Madrid, España: Ediciones Mundi-Prensa.

Shanks, A. L. (2002). Interanual tides and the biology of continental shelf waters. In J. C. Castilla \& J. L. Largier (Eds.). The oceanography and ecology of the nearshore and bays in Chile. Proceedings of the International Symposium on Linkages and Dynamics of Coastal Systems: Open coasts and embayment (pp. 3-27). Santiago, Chile.: Ediciones de La Universidad Católica de Chile.

Sharov, A. F., Volstad, J. H., Davis, G. R., Davis, B. K., Lipcius, R. N., \& Montane, M. M. (2003). Abundance and exploitation rate of the blue crab (Callinectes sapidus) in Chesapeake Bay. Bull. Mar. Sci., 72(2), 543-565.
Smith. L. D. \& Haines, A. H. (1991). The effet of cheliped loss on blue crab Callinectes sapidus Rathbun foraging rate on soft-shell clams Mya arenaria L. J. Exp. Mar. Biol. Ecol., 151, 245-256. https://doi.org/10.1016/0022-0981(91)90127-I.

Soto, L. A. (1980, November). Decapod crustacean shelf-fauna of the Campeche Bank: fishery aspects and ecology. Ponencia presentada en Proceedings of the Gulf and Caribbean Fisheries Institute, Fort Pierce. EE. UU.

Sponaugle, S. \& Lawton, P. (1990) Portunid crab predation on juvenile hard clams: effects of substrate type and prey density. Mar. Ecol. Prog. Ser., 67, 43-53.

Taissoun, E. (1969). Las especies de cangrejos del género Callinectes (Brachyura) en el golfo de Venezuela y lago de Maracaibo. Bol. Cent. Inv. Biol., 2, 1-103.

Türeli, C., Miller, J., Gundogdu, S. \& Yesilyurt, I. (2016). Growth and mortality of blue crab (Callinectes sapidus) in the north-eastern Mediterranean Sea. $J$. Fish. Sci., 10(2), 55-62.

Valentine, J. M., Walther, J. R., McCartney, K. M. \& Ivy, L. M. (1972). Alligator diets on the Sabine National Wildlife Refuge, Louisiana. J. Wildl. Manag., 36(3), 809-815. https://doi. org/10.2307/3799434.

Villasmil, L., Mendoza, J. \& Ferrer, O. (1996). Análisis de las estadísticas de captura y esfuerzo para la pesquería del cangrejo azul, Callinectes sapidus, en el lago de Maracaibo para el periodo 1973-1993. Ciencia, 4(4), 293-307.

Villasmil, L., Mendoza, O. \& Ferrer, O. (1997). Crecimiento y mortalidad del cangrejo azul Callinectes sapidus Rathbun, 1986, del lago de Maracaibo. Ciencia, 5, 7-15.

Villasmil, L. \& Mendoza, L. (2001). La pesquería del cangrejo Callinectes sapidus 
(Decapoda: Brachyura) en el lago de Maracaibo, Venezuela. Interciencia, 26(7), 301-306.

Vincent, G., Perry, H. \& Vanderkooy, S. (Eds). (2001). The blue crab fishery of the Gulf of Mexico, United States: a regio- nal management plan. Ocean Springs, Mississipi, EE. UU.: NOAA.

Williams, A. B. (1974). The swimming crabs of the genus Callinectes (Decapoda: Portunidae). Fish. Bull., 72(3), 685-798. 